\title{
THE DOCTRINE OF PRIMARY AND SECONDARY SENSORY ELEMENTS. $\left(\mathrm{I}^{1}\right)$.
}

BY DR. BORIS SIDIS,

Brookline, Mass.

I.

The theory of perception is fundamental both in normal and abnormal psychology. All mental activities are intimately related with the process of perception. Our wills, our thoughts and our feelings relate to our experience of the outer world of things. Biologically regarded, the percept is of the most vital importance, inasmuch as it forms the medium between the individual and the outer environment; psychologically, the percept reflects the external world and mirrors the conditions of life to which the given organism has to adjust itself. In fact, the percept may be regarded as the coin possessing the value of the external environment. In this respect we cannot help agreeing with Professor Baldwin's statement: "The theory of perception is perhaps the most important as well as the most difficult problem in psychology. The interpretation of the higher processes of mind rests upon it and it underlies the body of our general philosophy. The great philosophies of the world take their rise from initial differences in the method of construing perception."

In abnormal psychology the theory of perception is of the utmost importance, both from a theoretical and practical standpoint. Illusions, hallucinations, dream states, subconscious states, many states of dissociation depend for their explanation on the analysis of the process of perception. In one of $\mathrm{my}$ papers on hallucinations, published in this Review, I have developed a theory of perception, a theory which may be characterized as the doctrine of primary and secondary sensory elements. This doctrine is based on a close analysis of the

1 The MS. of this article was received November I, 1907. - Ed. 
normal process of perception and is substantiated by observations and experiments of abnormal mental life.

Before however we state our view of perception it may be well to make a review of what the principal psychological authorities teach on the subject.

James Mill in discussing perception tells us: "The colors upon a body are different, according to its figure, its shape, and its size. But the sensations of color and the sensations of extension, of figure, of distance have been so often united, felt in conjunction that the sensations of the color are never expe. rienced without raising the ideas of the extension, the figure, the distance in such intimate union with it, that they not only cannot be separated, but are actually supposed to be seen (italics are mine). The sight, as it is called of figure, or distance, appearing, as it does a simple sensation, is in reality a complex state of consciousness a sequence in which the antecedent, a sensation of color, and the consequent a number of ideas are so closely combined by association that they appear not one idea, but one sensation."

Sully defines perception as a mental act that "supplements a sense impression by an accompaniment or escort of revived sensations, the whole aggregate of actual and revived sensations being solidified or integrated into the form of a percept.' The revived sensations are equivalent to James Mill's associated ideas and images. We shall point out later the confusion which generally prevails among psychologists and psychiatrists, when they talk indiscriminately of revived sensations and ideas regarding the two as identical.

Höffding describes the process of perception "as the fusing of a reproduction and an actual sensation. The percept is thus conceived as compounded out of a representation and a sensation."

Taine tells us that "Images associated with the sensations of the different senses, especially with those of sight and touch constitute acquired perceptions."

Wundt regards the percept as a psychical compound of ideas or of revived sensations or images. In that respect his analysis differs but little from that of other psychologists who regard 
the ideas, images and revived sensations as identical elements going to form the associated whole or psychic compound, the percept.

Külpe speaks of 'centrally excited sensations' regarding them as the ideas and the images of the psychologists and psychiatrists and tells us that he avoids the use of 'ideas.' As far as perception is concerned he closely follows his master Wundt and talks of psychic compounds, of sensations and centrally excited sensations which really are identical with the old fashioned ideas and images.

Titchener follows closely Wundt and Külpe and regards the 'percept as a compound, or a complex of sensations,' of peripheral and of centrally initiated sensations. In order to be explicit he hastens to tell us that there is no fundamental difference between the perception and idea. "It is customary to speak of perception, when the majority of the simple processes in the complex are the result of stimulation of a sense organ, $i$. $e$., are peripherally aroused, and of idea when the greater number are the result of an excitation within the brain cortex, $i . e$. , are centrally aroused. If I have a table before me and my eyes open I am said to perceive the table; if I close my eyes and think of what I saw, to have an idea of a table. But we have seen that the sensations aroused centrally do not differ as psychological processes from those aroused peripherally." This statement put in such an explicit form brings out clearly what may be designated as the psychologist's fallacy. The fallacy becomes specially apparent in the domain of abnormal psychology.

Baldwin with his characteristic breadth of comprehension puts the subject of perception on a wide basis: "Perception is the apperceptive or synthetic activity of mind whereby the data of sensation take on the forms of representation in space and time; or it is the process of the construction of our representation of the external world." Baldwin does not commit himself to the ordinary fallacy current among psychologists.

Similarly James with his genius for psychological insight tells us: "The consciousness of particular material things present to sense is nowadays called perception." And again 
"Perception thus differs from sensation by the consciousness of farther facts associated with the object of the sensation." He tells us further: "We certainly ought not to say what usually is said by psychologists and treat the perception as a sum of distinct psychic entities, the present sensation namely, plus a lot of images from the past, all integrated together in a way impossible to describe. The perception is one state of mind."

We thus see that most of the psychologists regard the percept somewhat in Spencerian terms as being made up of presentations and representations, or as Spencer puts it as being ' partly presentative and partly representative.' In other words the percept is a compound of sensations and images, a synthesis of peripherally induced sensations and of images, or of ideas centrally excited. One principle underlies the current theory of perception, variously phrased by different psychologists, and that is the identification of ideational and sensory processes.

The identification of ideational and sensory processes may be traced to Spinoza when he tells us in his Ethics, Prop. XVII., note, "The modifications of the human body, of which the ideas represent external bodies as present to us, we will call the images of things" and then in another place of Part II., Prop. XLIX., note, "In order to illustrate the point let us suppose a boy imagining a horse and perceiviug nothing else. Inasmuch as this imagination involves the existence of the horse, and the boy does not perceive anything which would exclude the existence of the horse he will necessarily regard the horse as present; he will not be able to doubt its existence, although he be not certain thereof. We have daily experience of such a state of things in dreams." The images, according to Spinoza, are equivalent to sensations and percepts, unless counteracted by the more intense peripheral sensations which thus become the 'reductives' of the image, a doctrine afterwards fully developed by Taine. I may add that Spinoza's view of dreams is repeated almost verbatim by the greatest psychological authorities, all uncritically giving their assent to the current fallacy that the image is but a weakened sensation and that the sensation is an intensified image. ${ }^{1}$

'In my experimental work on sleep and dreams, soon to be published, I take up the subject in detail. 
This theory of images and perception is perpetuated through Hobbes, Locke, Hartley, Hume, James Mill down to our times.

Hobbes in his terse English puts it: "Imagination therefore is nothing but decaying sense and is found in men and many other living beings, as well in sleeping as waking."

Locke derives his 'ideas' from 'experience,' but his ' experience' is somewhat vague and broad, inasmuch as it flows from two fountain heads, - sensation and reflection. "Let us then suppose the mind to be as we say white paper void of all characters without any ideas, how comes it to be furnished? . . . To this I answer in one word from experience. . . . Our observation employed either about external sensible objects or about the internal operations of our minds, perceived and reflected on by ourselves is that which supplies our understanding with all the materials of thinking. These two are the fountains of knowledge from whence all the ideas we have or can naturally have, do spring." Perception is used by Locke in a broader sense than what it is understood at present, as he uses perception for sensory experience as well as for the introspection of higher mental processes. He tells us, however, that in either case " the mind has a power to revive perceptions which it has once had, with this additional perception annexed to them that it has had them before." Locke evidently entertains the view that sensations can be revived as original sensory experience and that the revived ideas do not differ, except for the addition of pastness, from the original ideas derived from the great source of sensation.

When we pass to Hartley and Hume the identification of sensation and idea is set forth with great explicitness. In fact, it is taken as the fundamental principle of their psychological systems. Thus Hartley postulates in his eighth proposition that "Sensations by being often repeated leave certain vestiges, types or images of themselves which may be called simple ideas of sensation" and correspondingly we have that "sensory vibrations by being often repeated beget in the medullary substance of the brain a disposition to diminutive vibrations which may be called vibratiuncles and miniatures corresponding to themselves 
respectively." The vibratiuncle is the physical substratum of what we experience as an idea and is a copy of the original vibration. The vibratiuncle is a weakened vibration and the idea is a weakened sensation.

Hume does not burden himself with Hartley's vibrations and vibratiuncles, but still at the basis of his system we find the same fallacious psychological principle. "All our ideas" he says " are copies of our lively perceptions or impressions." In other words, our sensations are lively impressions, while the ideas are only weakened perceptions, - the idea differs from the sensation only in intensity. There is no qualitative difference between sensation and idea. Ideas belong to sensory processes and do not differ as such from sensations. This view has since become the heritage of current psychological theories.

\section{II.}

As in many other sciences, especially the ones of the purely mental variety, a good deal in psychology is traditional such for instance are the tripartite and bipartite division of the mind or the various classifications of the mental activities. Of course, classifications as well as theories have their important function in science, but they should not be permitted to become a bed of Procrustes to the guests whom they shelter.

It may sometimes be well to disregard established principles, classifications and time-honored traditions and study the facts from a somewhat different standpoint. We may then possibly see the facts in a new light and realize aspects and connections which are hidden from the customary view of the phenomena.

Suppose we take a mental cross-section of a moment of perceptual consciousness in the very act of formation of a percept. The whole perceptual moment may be said to be spread out before our mental gaze. We find sensory elements of a relatively intense character. Certain sensory elements stand out first and foremost in consciousness, they are the very first to arrest the mental gaze and keep it steadily fixed on themselves. In the same view however we can also discern other elements, not so prominent, though equally sensory which, on account of their 
lack of prominence, appear to be of a subordinate character. The whole tone of the percept is given by the qualitative aspect of the prominent elements which seem to guide and form the organization of the percept.

The general plan of the structure of the percept may be compared to that of the cell. A close examination of the cell reveals the presence of a central element, of a nucleus surrounded by cytoplasm with its meshwork, the cytoreticulum. The nucleus forms the central and important structure having the functions of assimilation and reproduction. The nucleus and cytoplasm however are intimately related; the modification of one affects the other. Both nuclear and cytoplasmic structures form one organized whole, one living cell. Similarly in the percept we find a group of sensory elements which constitute the nucleus and a mass of other sensory elements, possibly the main mass, forming the tissue of the percept. The nuclear elements are more intense and appear to be predominant in the total mental state, - both however are intimately connected and go to form the living tissue of the percept. The nuclear elements of the percept have the lead and seem to possess the organizing, the fermenting power to assimilate the mass of subordinate elements and have them transformed into one unified organic whole. The slightest modification in the structure and function of the nuclear elements brings about a change in the total cytoplasmic mass of the percept, giving rise to a different structure, to a different percept ; and again, modifications of the cytoplasmic mass, so to say, affect the formation of the nuclear elements often resulting in a different percept. It requires however quite a considerable change in the subordinate elements to bring about a change in the percept; while the slightest modification of the nuclear elements, whether in quality or interisity, will often bring about a fundamental transformation of the percept. The nuclear elements may be regarded as the sensitive, as the vital point of the perceptual system. We cannot displace nor can we modify the nucleus of the percept without profoundly modifying or even completely destroying the life existence of the percept.

We may point out here a very important aspect of the percept, 
an aspect which has been neglected by the older psychologists, but which is now being more and more emphasized by the younger psychologists who lay more stress on the functional and biological side of mental life. Like the life of all organized beings, the life existence of every psychic state is for some reaction, for some adjustments to the conditions of the external environment. In the struggle for existence the animal organism must on pain of death be adjusted to the objects of its external world. Now the central, nuclear, sensory elements awakened by external excitations give the cue for the reaction, they form the sensitive organization for the release of motor energy in definite directions, they signify a definite object to which correspond definite motor tendencies with final reactions of adjustment. To the mouse the cat is not an object of contemplation or an object of observation, on account of its sensory effects, - the cat is an object to run away from. To the dog a cat is not an object of beauty, but something to be run after. The sensory stimulations coming from the 'that' which is mouse is for the cat something to be on the alert, to jump after and to attack. In fact the lower we descend in the scale of animal life, the more prominent do the motor reactions become. Where life is predominantly of the instinctive type, the motor side of consciousness is more apparent. The fly attracted by the scent to deposit its eggs in decomposed meat; the wasp that strikes the caterpillar in definite places paralyzing its nervous system, thus preparing food for the coming larva ; the newborn infant starting to suck, when put to the breast - are good examples of motor reactions in response to sensory stimulations coming from external objects. A definite sensory stimulus is the trigger which releases a definite set of motor reactions. The fly, the bee is hardly conscious of the sensory characters of the honey; it is more likely that the sensory stimulations of the honey release the appropriate reaction of flying towards it. The bright colors of flowers developed in the course of natural selection for the fertilization of plants serve the same purpose; they awaken definite responses useful both to plant and insect, as it is hardly probable that the insects are primarily attracted by the beautiful coloring of the flowers. The visual stimuli awakening defi- 
nite sensory elements may be regarded as central and nuclear which in turn serve as a highly sensitive trigger to release definite systems of motor reactions. The effect is somewhat similar to that of the moth attracted by the flame, - the flame acts as a peripheral stimulus giving rise to sensory elements which form the sensitive trigger in the release of the reaction of circling around the flame, in spite of the harmful results. The moth reacts to bright objects in going towards them, but this particular bright object, the flame, has not been provided for in the motor adjustments of the moth, hence the lack of adaptation, the going to the danger, instead of flying from it.

So apparently insignificant is the sensory side and so predominant is the motor side with its almost mechanically fatal reactions, that some physiologists put the whole mechanism of excitation and reaction in the lower animals under the category of tropisms, which may be positive or negative, according as the animal goes to or from the particular stimulus. The sensory side is denied, the whole affair is simply a delicate chemical reaction such as the chemotaxis of leucocytes in the phenomena of phagocy tosis observed in inflammations and bacterial invasions, or what is still simpler as the phenomena of heliotropism observed in the case of plants. This purely mechanical or chemicophysiological view may be crude and far fetched in the case of lower animals, but it brings out strongly the predominance of the motor reaction in response to definite sensory excitations. The motor attitude of the animal towards the excitations of the external environment constitutes the predominant part of its objective world. The reactions with their sensory-motor effects are part and parcel of the total percept. Sensori-motor life gives reality to the world of objects. The spatial, the resistant, the material character of objects depends on our motor reactions which give content and reality to the world of things. Activity gives the sense of 'physical' reality, the sense of material actuality, or of what is regarded as 'the really real.' In other words sensory-motor reactions with consequent kinæsthetic sensations may be regarded as constituting the very essence of the real, external, material world, - the world of external, material objects. 
III.

The percept as we have pointed out forms one organic whole, the constituent elements are firmly integrated into one living organization. In other words, just as the organism is not simply an integrated compound of cells, tissues and organs, but all those lower units go to form the higher living unit, the life of the organism as a whole, so we may say that the sensory elements are not the same as the percept, they are analytically found, on the autopsy of the percept, - the sensory elements are the lower units that help to form the higher unit, the living percept. From a scientific standpoint, as the resuit of psychological analytical dissection, the sensory elements going to make up the psychic compound, the percept may be regarded as different from the total synthesis with its characteristic living activity and its peculiar form of perceptual consciousness.

The constituent elements of the percept are not of the same definiteness and intensity. The central nuclear elements stand out more distinct, more definite and consciouness lights them up with more power and intensity. They are like the mountain peaks - when glade and valley and mountain side are still immersed in darkness, the rising sun greets the mountain tops and plays and caresses them with its rays; when again the shades of evening begin to flit and gather over vale, ravine and gulch, the rays of the setting sun long linger on the peaks taking of them their last farewell. The central nuclear elements are in the focus of consciousness, - they are the first to be met by the glance of the mental eye and are the very last to be left by it. Consciousness plays with its search-light on the nuclear sensory elements. The central nuclear elements are intense, distinct and definite, while the subordinate elements are of far less intensity, are often quite indistinct, are, so to say, on the fringe of consciousness; in fact, may even be entirely subconscious. And still indefinite, indistinct and submerged as those subordinate elements are, they form the main content of the percept, giving it the fullness of reality.

The nuclear elements form the cue of the total reaction, thus standing for the particular object, forming the reality of the percept for the organism. No wonder then that the cue, 
though it may be the smallest portion of the percept, none the less forms for the organism the most vital, the most significant as well as the most constant part of the percept. The attitude, the total reaction of the organism depends on the slightest difference in the cue, on the slightest change of the nuclear elements, since the apparently slight modification may often prove of great significance to the life existence of the organism, - it may be a matter of life and death. The nuclear elements constitute the signal, the sensitive trigger for the release of definite reactions towards the changes of external objects. Hence the nuclear elements come to signify, in fact, to constitute the essence of the percept.

A change of the subordinate elements of the percept does not matter so much as the slightest modification in the quality or even in the intensity of the signal. This, of course, does not mean that the subordinate sensory elements are not psychologically and biologically of the utmost consequence to the organism, but they are not of that immediate importance as the focal nuclear elements appear to the consciousness of the organism. The nuclear elements as signal focus the interest of the animal. We can well realize their vital importance, if we consider that the nuclear elements are the flag which indicates friend or enemy, war or peace, life or death.

\section{IV.}

If we regard the percept statically, we may describe it figuratively as a psychic compound, the union of the elements having somewhat the character of a chemical combination. A new compound is formed possessing qualities of its own, different from those of the constituent elements. The sensory characteristics are profoundly modified in the synthesis, so much so that they cannot be directly discerned and can only be discovered by patient analysis. The elements do not exist freely, they are bound up in one indissoluble union of the percept. It seems, as if different qualitative states arise in the union, the qualities of the elements appearing, as if transformed by the effected synthesis.

The percept forms a new compound in which the component 
elements are disguised and transformed by the qualitative aspect of the central elements. The subordinate elements become adapted to the active nucleus and come out in the compound with sensory characteristics foreign to their nature. In the process of synthesis the subordinate elements become transmuted and assume the sensory characteristics of the nucleus. To analyze the various elements out of the synthetized percept, the central elements must be shifted, - the subordinate elements must be made focal, giving rise to new percepts, but at the same time making it possible to pass in review the various elements. In other words, the elements become revealed in proportion as we make of them signals, in proportion as they become significant of the total percept with its sensori-motor reactions.

The nuclear elements are the most pronounced, the most prominent, as far as saturation of sensory quality is concerned. They have so much of their peculiar sensory quality that they diffuse it into the other elements, - the subordinate elements appear under the sensory form of the nucleus; they become assimilated by the nucleus and are saturated with its sensory coloring. This holds true not only in regard to saturation, but also in regard to sensory brightness. The central elements possess a sensory brightness far in excess of other elements and hence they shed their sensory light on the more obscure, though no less important sensory elements. What however they illumine is not so much the peculiar sensory characteristics of those elements, but their own coloring with which they have saturated the total percept.

The force of the central elements lies specially in the emotional or affective tone with which they are pervaded. They arouse an attitude towards the external world in general and to the special object in particular, Taine would call it a tendency. The individual is stimulated by those nuclear elements and his whole attention is going out in direction to the object that has excited them. The whole organism is invaded by the subtle influence of the nucleus giving rise to definite sensori-motor reactions, intensifying the affective state which permeates the perceptual consciousness. The affective state of the percept is not always obvious in cases of fleeting percepts, but it becomes 
manifest, when the central elements become temporarily fixed, the stress and strain of consciousness tending in one direction. The very changes occurring in the flickering intensity of the nuclear elements tend to sharpen the situation, to enliven the interest, strain the attention and be all agog so to say. The cat getting a glimpse of a mouse, or the dog catching sight of the cat may be taken as good illustrations of the affective states present in perceptual consciousness. The nuclear elements are the ones that are specially charged with affective or emotional states.

Biologically regarded, we can well see the importance of the central nuclear elements, the necessity of their standing out in consciousness as more prominent and more intense than the rest of the sensory elements. Constituting the signal, they come to be the most significant part of the percept, for they announce what ' that' is, they present the object, friend or foe, something to welcome or something to flee from. The central nuclear elements thus come to present objective reality, they safeguard the individual, they are the safety as well as danger signal. The more delicately differentiated those safety-danger signals are, the more protected the individual is in the struggle for existence. The more sensitive the individual becomes to the least difference of the nuclear elements, the better adjusted will he be to the conditions of the external environment and the better will be his chances in the process of survival of the fittest.

This brings us to the purposiveness of the percept. One of the important characteristics of the biological process is the final cause, the purpose which leads to the preservation of that process, to the preservation of the individual. We should therefore expect that in the psychic process which is the most highly developed biological process, purposiveness will be one of the most important traits. In the course of phylogenetic and ontogenetic evolution some sensory elements, the ones to which the organism is more sensitive, will be selected and become the indicators of the total percept, they will become the index or better to say the pain-pleasure flag, the safety-danger signal. The central elements will thus be the most prominent, the most intense for that particular state of perceptual consciousness. The 
nature and character of the elements will vary with the organizations of the species and the individual. The dog will become more sensitive to variations of his olfactory sensations, while man will show marked sensitivity towards delicate differences of his visual sensory elements.

The great sensitivity of the nuclear elements is significant in so far as they lead to better adaptation and to more successful reactions. It is not of any consequence for the cow to gaze at the stars, for the pig to observe the phases of the moon, but it is a matter of importance for them to perceive any signs of food or the approach of a beast of prey. The heavenly bodies are non-existent for the brutes, because of lack of all reactions of adaptation, while food and predatory beasts are easily detected, because of the vital reactions bound up in the elements of the percept of which the nuclear elements form the signal. It is on account of the vital reactions that the perceptual nucleus plays such a prominent part and takes the lead of all the other elements. As I have pointed out in a former paper: "Every psychic state is for some reaction and that sensory element which gives the cue for the formation of the psychomotor elements, leading to some given reaction is, for the time being, the center, the nucleus of the total state."

\section{V.}

If we inspect the percept more closely, we find that there is some important difference in the character of the various constituent sensory elements. The central elements forming the nucleus of the percept are given directly by the sense-organ stimulated by its appropriate sensory stimuli, while the subordinate sensory elements are given indirectly, - they cannot be traced to appropriate sensory stimuli exciting those particular sense-organs on the activity of which those subordinate elements depend for their manifestation. In perceiving the lump of ice I can see the color, the size, the volume, the smoothness, the transparency, the distance and even the weight and coldness. Now what I can see directly is only the color, transparency, size, as given immediately by the stimulated sense-organ, by the visual sensations and image on the retina. Whence then come the 
rest of the sensory elements so distinctly experienced? They are not memory images, - they have the same sensory characters as the elements given by the direct impression of the sense-organs. It is not that on perceiving a certain transparent object we remember its volume, its distance, its smoothness, its resistance, we perceive all that in sensory terms. They are not images, ideas, or representations - they are sensations. The central sensory elements may be termed direct or primary, while the subordinate elements may be termed indirect or secondary. The percept then may be regarded as consisting of two classes of elements of sensations, the primary and secondary sensory elements. ${ }^{1}$

The secondary sensory elements are not images, nor ideas, nor representations, different terms employed for the same state by various writers, the secondary elements of the percept are essentially sensations. Now sensations are qualitatively different from images, ideas or representations. The image of a light does not shine, the idea of a voice does not sound and the representation of a perfume does not smell. A sensation or presentation as it is sometimes termed differs from an image or representation qualitatively, fundamentally. The sensation or presentation is given as immediate experience, while the image, the representation is essentially mediate, it is a mental substitute for the immediate experience of the sensation. The idea bears the same relation to the sensation as the photograph bears to the original, or rather as a symbol to the thing it represents. Ideas, images, representations symbolize sensations, but they are not sensations. A sensory process is fundamentally different. A sensation is not an intense idea, nor is an idea a weak sensation. Ideas differ far more qualitatively from sensations than visual sensations, for instance, differ from olfactory sensations. There is not a particle of evidence to substantiate the view that ideas or images are copies of sensations in the sense of being weak sensations or 'centrally excited sensations.' There is nothing of the sensory in the idea. The weakest sensation cannot compare

\footnotetext{
${ }^{1}$ It may be well here to point out that the doctrine of primary and secondary sensory elements advanced by me has nothing in common with the primary and secondary qualities of the older psychologists.
} 
with the most vivid representation. The laboratory experiments on that subject (Münsterberg and Külpe) are inconclusive as they either deal with incompletely perceived impressions, or with minimal sensations. In either case the percept is incomplete and uncertain. Külpe himself is forced to admit that ideas or centrally excited sensations as he terms them "cannot be regarded as simple revivals of peripherally excited contents, if only for the reason, that their remaining attributes are very rarely indeed identical with those of perception." He then goes on making a fatal admission: "The most striking evidence of disparity is perhaps afforded by intensity. . . I I is only in special cases that centrally excited sensations can rise from their accustomed faintness to the vividness of sense perception. We then speak of them as hallucinations (?); and they enter into a disastrous competition with the real material of perception, completely transcending the boundary line which so usefully divides it from the material of imagination." Külpe admits that there is no intensity to the image, that there is no variation in ' intensity' of images, an 'attribute' characteristic of percepts. Psychologically regarded this in itself shows the qualitative difference between image and percept.

Ideational and perceptual processes cannot be identified. The two are qualitatively different: the sensation has intensity, the image lacks it. We shall discuss later on the main differences of sensation and image, meanwhile we may point out the differences in brief: (a) A sensation has intensity, an image totally lacks it ; $(b)$ the image is a reproduction or rather a representation, a symbol of a sensation, but no sensation represents another; a sensation unlike an image, is not mediate, but immediate experience ; $(c)$ a sensation bears the mark of externality, an image lacks it; finally $(d)$ a sensation cannot be called up at will, while an image is independent of peripheral stimulations of external objects and is usually under the control of the will. No sensation differs so much from another as the image differs from its corresponding sensation.

To refer as Külpe does to a hallucination as an intensified image is to reason in a circle and at the same time to be in sad contradiction with facts. A hallucination may be regarded as 
a fallacious percept, but it is not on that account an image; a hallucination is a percept and is essentially sensory in character. The fact of a percept being fallacious does not in the least imply that it is 'imaginary' and not sensory. The ambiguity of the word 'imaginary' has not a little contributed to the psychological fallacy helping towards the confusion of image and sensation. 'Imaginary' is used in the common sense meaning not corresponding to any external reality, or in the psychological sense of consisting of those internal events or processes known as images or ideas. Now 'imaginary' used in the sense of lack of an external object by no means implies the psychological sense of consisting of images. A hallucination is commonly said to be imaginary in the sense of not having an objective reality, but we have to prove yet that it consists of images. The theories of illusions, hallucinations as well as of dream states and hypnotic hallucinations are vitiated by that fundamental psychological fallacy. As a matter of fact hallucinations are not made up of images, but of sensory elements, while on the contrary hypnotic hallucinations are not made up of sensory elements, but of images. In a former paper of mine I have pointed out that hallucinations are not due to 'images' but to actual sensations, that, psychologically regarded, hallucinations do not differ in their make-up from ordinary percepts. Ideas and images are not possessed of magic virtues and with all the fancy work about them, they cannot display sensory qualities. The image or idea is that bloodless, shadowy, fluttering affair which can no more attain the life of a sensation than a written letter can attain the power of sound. Had it been otherwise the world would have been a large asylum for images to play their pranks in. ${ }^{1}$

${ }^{1}$ We may quote Stout as one of the few psychologists who seem not to accept the current psychological doctrine. In his 'Analytic Psychology' he tells as 'that complex perception does not consist in a given impression reviving a cluster of faint images of previous impressions.' And again "impressional revival does not in the least countenance the theory that ideas are merely faint revivals of impressions. On the contrary, it tends strongly in the opposite direction. It shows that a revived impression is itself an impression, and not an idea." In his 'Manual of Psychology' he says 'that at bottom the distinction between image and percept is based on a difference of quality.' And again, "percepts and images possess a relative independence. This can be acconnted for, if we suppose that the nervous tracts excited in perceptual process are not wholly coincident with those excited in ideational process." 
The elements of the percept are not ideational, not imaginary, they are essentially sensory. The perceptual elements are synthetized into one percept. To take our stock example, the ice. The lump of ice is experienced as one object with many qualities each of which furnishes respectively its sensory quota towards the formation of the whole of the perceptual experience. We see, we perceive the hard, heavy, smooth, resistant body of ice, - all the elements have alike the intensity of sensation. The hardness, the smoothness, the bodily resistance are perceived by the visual sense and are visual, but as such they of course differ from the sensations experienced by their appropriate sense organs, as when for instance the same sensations are given by touch or by muscular and kinæsthetic sensations. Those muscular and tacto-motor sensations appearing as visual are not memory-images, but they are actual sensations, they are secondary sensations; they are secondary sensory elements which give the fullness of content to the percept having visual sensory elements as its nucleus. Unlike memory-images, secondary perceptual elements have the immediacy of sensational experience. Remembered sensory qualities are not immediate experiences given in the object of perception.

If we turn to pathology, we find that cases closely confirm our view. In certain mental diseases the patient can perceive the various qualities, although he cannot represent them to himself. In other cases the patient can clearly and vividly represent objects in all their details, but he cannot perceive the objects, when directly confronted with them. Clinical cases, even if we exclude all facts from introspective analysis, clearly point to the qualitative difference of image and sensation irrespective of the assumption of localization, - they may be due to the function of different brain structures, or to different processes of the same brain structures. In the light of recent research it is more likely that the neuron structures underlying ideational processes differ from those subserving sensory processes. Whichever view however we entertain in regard to the anatomical structures all the facts go to prove that image and sensation are qualitatively different psychic events.

The percept is not ideational, but sensory. There are no 
memory-images in perceptual consciousness, although the latter may be closely associated with ideational processes. Such ideas however are on the fringe of the perceptual consciousness and do not constitute the essence of the percept. The percept consists of sensory elements, primary and secondary. The primary elements are initiated directly by incoming peripheral stimulations, while the secondary sensory elements are brought about indirectly, through the mediacy of the primary elements, the secondary elements themselves being really derived from senseorgans others than the one directly stimulated by the peripheral excitation.

If the percept is visual and $V$ stands for the visual physiological processes, $A$ for the auditory, $O$ for the olfactory, $M$ muscular, $K$ kinæsthetic, $T$ for tactual physiological processes ; then let $V_{1}, M_{1}, O_{1}, K_{1}, T_{1}$ stand for the primary sensory elements; and let $V_{2}, O_{2}, M_{2}, K_{2}, T_{2}$ stand for the secondary sensory elements, then the total percept may be represented by the formula $V_{1} \mathrm{O}_{2} M_{2} K_{2} T_{2}$. Since all the other elements appear in the visual percept under the visual aspect we may represent the percept by the formula: $V_{1} M_{2}^{v_{1}} O_{2}^{v_{1}} K_{2}^{{ }_{1_{1}}} T_{2}^{v_{1}}$. The secondary sensory elements, though forming the main content of the percept, are apparently of a visual nature and still they really belong to qualitatively different realms of sensations. This clearly reveals their origin and nature: the secondary sensory elements are not visual, but they become so by being initiated through the visual sense. In other words, secondary sensory elements are not peripherally initiated. Are they then centrally excited sensations? No. They can only be induced by an external stimulus. But that external stimulus must act indirectly, through another sense-organ. In stimulating a senseorgan we not only get sensory elements characteristic of that particular sense, but also sensory elements belonging to other sense-organs which have not been stimulated. What really takes place is this : the external excitation acting on a particular sense-organ produces its appropriate sensations, but the peripheral physiological process diffuses or rather to say gets irradiated along other neurons of other sense structures, awakening their appropriate sensations Such sensations, not being directly, 
but indirectly peripherally initiated should be regarded as secondary sensations.

VI.

The phenomena of secondary sensations are well known in psychological literature. Some psychologists following the general fallacy of confusing image and sensation describe vivid images succeeding sensations under the category of secondary sensations. Barring such confusion we may say that the pure phenomena of secondary sensations are essentially sensory in character. When a sensation due to the stimulation of a peripheral sense-organ, instead of being followed by a train of association of ideas is followed by another sensation belonging to the domain of another sense-organ, the phenomenon is known as that of synæsthesia or of the secondary sensations.

One image or representation relating to a sensation of one sense-organ may be associated and bring in its train of associations any other image relating to any other sensation of any other sense-organ. The series of ideas or images will be a reproduction of stimulated sense-organs with their accompanying sensations, the ideas running parallel to the original psychophysiological processes, somewhat on the Spinozistic principle of ' Ordo et connexio idearum idem est ac ordo et connexio rerum.' And again in other cases, when not reproducing a previous series of sensory experience, the series of associated images may be more irregular and apparently capricious - a process usually described as the work of fancy, or imagination. A sensation or image then may be followed by any series of images without the intermediacy of external excitations and peripheral physiological processes, but a sensation cannot be followed by a series of sensations without the intermediacy of external stimulations. A sensation can only be initiated by its own appropriate stimulus and by its own specialized peripheral physiological processes. The smell of a rose will not by simple association give rise to a series of sensations of touring in an automobile, nor will the eating of a beefsteak give rise, through association, to the hearing of a symphony. In other words, there is an internal association of images or ideas, but there is 
not an internal association of sensations. Images once born can be reproduced endlessly and at will, sensations die almost immediately after they are born and must be renewed every time ander the same conditions of external stimulations. Briefly stated, there is memory for images, but not for sensations. Sensations are independent, images are interconnected.

If we represent sensations by $A, B, C, D$ and symbolize images by $a, b, c, d$, the $A, B, C, D$ have no relations to one another, but each one bears a definite relation to each corresponding image, $A$ to $a, B$ to $b, C$ to $c, D$ to $d$ and so with the rest of the series. Sensation $A$ will arouse image $a$ which in turn may arouse the whole train of images $b, c, d$, but $A$ cannot give rise to any of the sensations $B, C, D$. The image series $a, b, c, d$ can be reproduced at will, in fact after a series of repetition the whole chain of links may rattle off against will, but nothing of the kind occurs in the case of sensations. Sensations do not form links in a chain which becomes automatic after many reproductions. Repetition of sensations does not form associated series; sensations maintain their independence.

The difference between image and sensation in respect to association is, psychologically regarded, apparently flawless. Unfortunately as it is usually the case with flawless generalizations and descriptions of phenomena observed under normal conditions, there is an ungracious ' abnormal' that refuses to fall into line. There are cases apparently abnormal from the psychological standpoint, cases which refuse to be gathered into the normal psychological fold, the cases seem to run counter to all normal psychological introspection. The sensations seem to run riot, - instead of being linked with their respective images they really call up associated sensations, these are the so-called sound-photisms or light-phonisms and similar odd combinations. It is true the sensations are rather awkwardly associated. One sensation always calls forth only a particular sensation and no other one, and besides the called forth sensation does not belong qualitatively to the same domain with the one that has initiated it. It is also true that the sensations show their lack of sociable character by not entering into any association with any other sensation, and that unlike images no asso- 
ciative series can possibly be formed. Still the fact remains that a sensation can and does call forth another sensation. Evidently sensations can enter into associative bonds. Such psychic states appear uncanny and are regarded as abnormal. The phenomena are regarded as freaks belonging to the domain of pathology. Now curiously enough our study reveals the fact that what has been regarded as the pathological and exceptional turns out to be the ordinary and the normal. The stone which the builders neglected has become the corner stone. The exception has turned out to be the rule. Far from being the case that secondary sensations are rare and abnormal, they are quite common, since they constitute the very flesh and blood of the percept. Secondary sensations constitute the texture of the percept. The reason why they appear so strange is just because they are so common and so familiar.

The secondary sensation, when appearing alone out of its perceptual complex, cannot be recognized as the old familiar attendant belonging to the indissoluble retinue of the humdrum percept. Dissociated from its perceptual sphere the secondary sensation appears ghostly, hallucinatory. As a matter of fact the secondary sensation, hallucinatory and spooky as its manifestations are, constitutes part and parcel of perceptual experience. In fact, the main content of the percept consists of hallucinatory secondary sensations. Percepts and hallucinations are of the same grain. A percept is a hallucination with the primary nuclear sensory elements present, a hallucination is a ' real' percept with the primary sensory elements absent.

When secondary sensory elements become dissociated from the perceptual synthesis with the primary sensory elements, the elements, thus dissociated, not being related to any peripheral physiological process of their appropriate sense-organ, are regarded as central phenomena, as secondary sensations which are described as unusual, abnormal events of mental life. What, however, is abnormal is not the secondary sensation per se, but the fact of its dissociation. A dissociated secondary sensory element becomes manifested as a secondary sensation. 
VII.

Secondary sensations are free secondary sensory elements, dissociated from the perceptual aggregate into the synthetic unity of which they enter as important components forming the organic whole of the percept. When appearing isolated, secondary sensations are the simplest form of hallucinations which become more and more complex as the secondary sensory elements, dissociated from the primary elements, become manifested in complex systems. Hallucinations are systems of secondary sensations or of secondary sensory elements.

Sensory elements are, as a rule, not free, they usually appear as perceptual compounds, and this holds specially true of secondary sensory elements. When therefore dissociated from their perceptual compounds, they appear as ghosts of the 'real' percept, as hallucinations. To quote from a previous work of mine: "The integration of the groups and especially of the secondary presentative groups is not of that unmodifiable organic character. Around a nucleus formed by a group, or combinations of groups of primary elements, groups of secondary sensory elements become aggregated, and the total aggregate gives rise to a consolidated and unified system of groups, resulting in a percept. In perceiving the chair yonder only the visual sensations constitute the true sensory groups that form the nucleus of the percept. The other psychic groups that are crystallized round the percept, such as weight, resistance, volume, size, shape, distance are really visuo-tacto motor groups; they are largely tacto-muscular groups tinged by the sensory quality of the nucleus; they are tacto-motor groups sensorially visualized, seen indirectly. Though these secondary sensory groups are firmly integrated, still their integration is not of such a character as not to become disintegrated and rearranged into new systems of groups. Such a disintegration is no doubt effected with difficulty, but it is by no means impossible. The perceptual compounds, unlike the sensory, admit of decomposition into elementary primary and secondary sensory groups. The component elementary sensory groups can be experienced separately under different conditions and circumstances. We can close our eyes and walk up to the object of perception, say the chair, 
and thus experience the free muscular sensations of distance, or we may push our hand against the chair and experience the sensation of resistance, or take the chair in the hand and experience the sensation of resistance, or take the chair in the hand and experience the muscular sensations of weight and shape. The primary and secondary groups going to make up the percept can be isolated by withdrawing the organizing nuclear group of primary sensations, thus bringing about a disintegration of the p articular aggregate.

"If we inspect more closely this process of isolation, we find that the constituent secondary sensory groups are not really isolated, so as to stand out all by themselves. What actually happens in this seeming process of isolation is simply the formation of a series of new perceptual aggregates in which the particular sensory groups that are isolated and specially brought out become the nuclei, the foci. For in the perceptual aggregate it is always the character of the nucleus that is specially brought out, and it is the nuclear aggregate that tinges with its sensory color all the other aggregates. To revert to our previous example, to the percept-chair. In passing the finger over the chair, the touch may form the nucleus of the moment, but around this primary nuclear sensory group other secondary sensory groups, such as thermal and muscular sensory elements become organized to form the synthesis of the perceptual moment. If we try to find out the shape of the chair by a series of touches, we really form a series of percepts, the sensory nuclei of which are not visual, but tacto-muscular in their nature. A sensory group then cannot in reality appear in a purely isolated form." In other words, sensory elements appear in groups, ${ }^{1}$ and this holds specially true of secondary sensory elements or of secondary sensations. Secondary sensations, though present in every percept, rarely appear in isolation. The affinity of secondary sensory elements to run into compounds becoming synthetized with primary elements makes it

'James lays stress on this fact of grouping of sensory elements: " All brain processes are such as give rise to what we may call Figured Consciousness. If parts are irradiated at all, they are irradiated in consistent systems and occasion thoughts of definite objects, not mere hodge-podge of elements." 
difficult to observe them, except in the peculiar phenomena of synæsthesia and in the abnormal states of hallucination.

If secondary sensations are simple hallucinations, hallucinations are compound secondary sensations. As we have pointed out in a previous paper on hallucinations, a close examination of hallucinations shows them to be cases of systems of secondary sensations dissociated from their primary nuclear elements. In states of dissociation a peripheral stimulation with its physiological processes and concomitant primary sensory elements may become dissociated from systems of secondary sensory elements which alone stand out in consciousness as hallucinations. A close examination reveals the presence of some obscure pathological conditions which by irritation and by irradiation awaken secondary sensory elements givnig rise to full fledged hallucinations. 